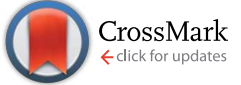

Cite this: RSC Adv., 2016, 6, 78720

Received 2nd June 2016

Accepted 15th August 2016

DOI: 10.1039/c6ra14327j

www.rsc.org/advances

\section{An ultra-low bandgap diketopyrrolopyrrole (DPP)- based polymer with balanced ambipolar charge transport for organic field-effect transistors $\dagger$}

\author{
Tao Jiang, ${ }^{a}$ Zhongyuan Xue, ${ }^{a}$ Michael Ford, ${ }^{\mathrm{b}}$ Jessica Shaw, ${ }^{\text {ac }}$ Xudong Cao, ${ }^{a}$ \\ Youtian Tao, ${ }^{\text {*a }}$ Yuanyuan $\mathrm{Hu}^{\star d}$ and Wei Huang*ae
}

\begin{abstract}
A new $A_{1}-A_{2}$ type polymer pDTDPP-TTF containing diketopyrrolopyrrole (DPP) and 2-ethylhexyl-3fluorothieno[3,4-b]thiophene-2-carboxylate (TTF) as the main electron-accepting building blocks has been synthesized via Suzuki cross-coupling polycondensation. The introduction of TTF as an additional electron-deficient unit significantly lowers the LUMO energy level of this DPP containing polymer to $-4.37 \mathrm{eV}$ which is beneficial for efficient electron injection and transport. The pDTDPP-TTF polymer shows an ultra narrow optical bandgap of $1.06 \mathrm{eV}$ with a broad absorption spectra covering the visible to near infrared region of up to $1300 \mathrm{~nm}$. Solution-processed OFET devices with PDTDPP-TTF as the active semiconducting layer have been fabricated with the configurations of both bottom-gate bottomcontact (BG-BC) and top-gate bottom-contact (TG-BC). The $\mathrm{BG}-\mathrm{BC}$ devices with $\mathrm{SiO}_{2}$ as a dielectric exhibit ambipolar behavior with a hole and electron mobility of $1.85 \times 10^{-4}$ and $3.55 \times 10^{-5} \mathrm{~cm}^{2} \mathrm{~V}^{-1} \mathrm{~s}^{-1}$, respectively. Improved OFET performance has been achieved in TG-BC devices by using PMMA as the dielectric; balanced ambipolar charge transport is obtained with a hole and electron mobility of $6.17 \times 10^{-3} \mathrm{~cm}^{2} \mathrm{~V}^{-1} \mathrm{~s}^{-1}$ and $3.13 \times 10^{-3} \mathrm{~cm}^{2} \mathrm{~V}^{-1} \mathrm{~s}^{-1}$, respectively. To the best of our knowledge, our work is among the very few examples of ultra-low bandgap semiconducting polymers with balanced ambipolar charge transport properties.
\end{abstract}

\section{Introduction}

Over the past few decades, organic field effect transistors (OFETs) based on semiconducting polymers have been extensively studied and developed for potential applications in flexible displays, ${ }^{1-4}$ radio-frequency identification (RFID) tag $\mathrm{s}^{5}$ and sensors ${ }^{6,7}$ etc., due to advantages such as low cost, large area, light weight and mechanical flexibility. Since the first report on polymer based OFET devices was published in $1986,{ }^{8}$ rapid progress with impressive charge-carrier mobilities (the typical figure of merit) has recently been demonstrated in this field., ${ }^{9,10}$

${ }^{a}$ Key Lab for Flexible Electronics and Institute of Advanced Materials, Jiangsu National Synergistic Innovation Center for Advanced Materials (SICAM), Nanjing Tech University, 30 South Puzhu Road, Nanjing, 211816, P. R. China. E-mail: iamyttao@ njtech.edu.cn; iamwhuang@njtech.edu.cn

${ }^{b}$ Materials Department, University of California, Santa Barbara, CA, USA

'Department of Chemistry and Centre for Plastic Electronics, Imperial College London, Exhibition Road, South Kensington, London SW7 2AZ, UK

${ }^{d}$ Cavendish Laboratory, University of Cambridge, JJ Thomson Avenue, CB3 OHE, UK. E-mail: yuanyuan.hu01@gmail.com

${ }^{e}$ Key Laboratory for Organic Electronics and Information Displays, Institute of Advanced Materials, Nanjing University of Posts and Telecommunications, 9 Wenyuan Road, Nanjing 210046, P. R. China

$\dagger$ Electronic supplementary information (ESI) available: Preparation of DPP monomer and Fig. S1. See DOI: 10.1039/c6ra14327j
In addition, ambipolar polymer semiconductors, in which both hole and electron charge carriers are demonstrated in a single active layer, have attracted considerable attention for their application in complementary metal-oxide semiconductor (CMOS) digital integrated circuits ${ }^{\mathbf{1 1} 12}$ and light-emitting fieldeffect transistors (LEFETs). ${ }^{13,14}$ Although several polymer semiconducting materials exhibited highly efficient ambipolar charge transport performance with mobilities in excess of $1 \mathrm{~cm}^{2} \mathrm{~V}^{-1} \mathrm{~s}^{-1},{ }^{10,15-17}$ the balance of ambipolar transport properties remains an important issue to solve.

In general, polymer semiconductors exhibit pronounced ambipolar charge transport behavior when a favorable energetic alignment is achieved such that both hole and electron transport is permitted. This is typically observed in polymer materials that possess a HOMO (highest occupied molecular orbital) energy level higher than $\sim-5.5 \mathrm{eV}$ (relative to the vacuum level) and a LUMO (lowest unoccupied molecular orbital) energy level lower than $\sim-4.0 \mathrm{eV} .{ }^{17}$ Therefore, only relatively low bandgap, at least below $1.5 \mathrm{eV}$ will allow for potential ambipolar transport. Presently, a general approach to achieve ambipolar conjugated polymer semiconductors is to design the polymer framework by involving alternating electron donor (D) and electron acceptor (A) building blocks. ${ }^{18,19}$ Highly efficient charge transport capability is often observed for these D-A type polymeric materials primarily due to the presence of efficient orbital 
overlap along the polymer backbone, which can facilitate intramolecular charge transport. ${ }^{20}$ Among electron acceptor building blocks, diketopyrrolopyrrole (DPP) has gained vast interest in the past few years for the construction of high charge mobility OFET devices, ${ }^{16,17,20-23}$ primarily due to its planar, conjugated, bicyclical structure and remarkable aggregating properties, which leads to strong $\pi-\pi$ interactions with polar carbonyl groups capable of hydrogen bonding. ${ }^{24-27}$ Currently, low bandgap DPP-based polymers have been widely studied in ambipolar OFET applications, several of which display some of the highest charge mobilities recorded to date. ${ }^{15,23}$ For instance, pTDPPSe-SiC5 (ref. 23) with an optical bandgap of $1.25 \mathrm{eV}$ exhibits the highest electrical performance in optimized conditions with high hole and electron mobilities of 8.84 and $4.34 \mathrm{~cm}^{2} \mathrm{~V}^{-1} \mathrm{~s}^{-1}$, respectively. However, most DPP-based D-A type semiconductors exhibit a significantly higher hole- than electron-transport performance. ${ }^{28-32}$ And as such DPP-based D-A type polymer semiconductors with balanced hole and electron charge transport mobilities are rarely reported. This is mainly due to difficulties for reaching a LUMO energy level below $-4.0 \mathrm{eV}$ which is necessary for facile injection and efficient transport of electrons in DPP-based D-A type semiconductors. ${ }^{22,33}$

In an effort to lower the LUMO energy level of DPP polymers, incorporation of another electron-deficient unit to the polymer backbone would be desirable. ${ }^{34,35}$ Herein, we designed and synthesized a new ultra low bandgap $\mathrm{A}_{1}-\mathrm{A}_{2}$ type polymer pDTDPP-TTF via a Suzuki cross-coupling polycondensation reaction between two electron-deficient units: $3,6-\operatorname{di}(2-(4,4,5,5-$ tetramethyl-[1,3,2]dioxaborolan-2-yl)thien-5-yl)-2,5-di(2-decyltetradecyl)-pyrrolo[3,4-c]pyrrole-1,4-dione (DTDPP) and 2-ethylhexyl-4,6-dibromo-3-fluorothieno[3,4- $b]$ thiophene-2-carboxylate (TTF) (Scheme 1). The polymer exhibited an ultra-narrow bandgap of $1.06 \mathrm{eV}$ and showed a broad and strong absorption spectrum extending to $\sim 1300 \mathrm{~nm}$. The strategy of molecular design on $\mathrm{A}_{1}-\mathrm{A}_{2}$ type DPP polymer successfully enables the balanced ambipolar behavior of pDTDPP-TTF in solution-
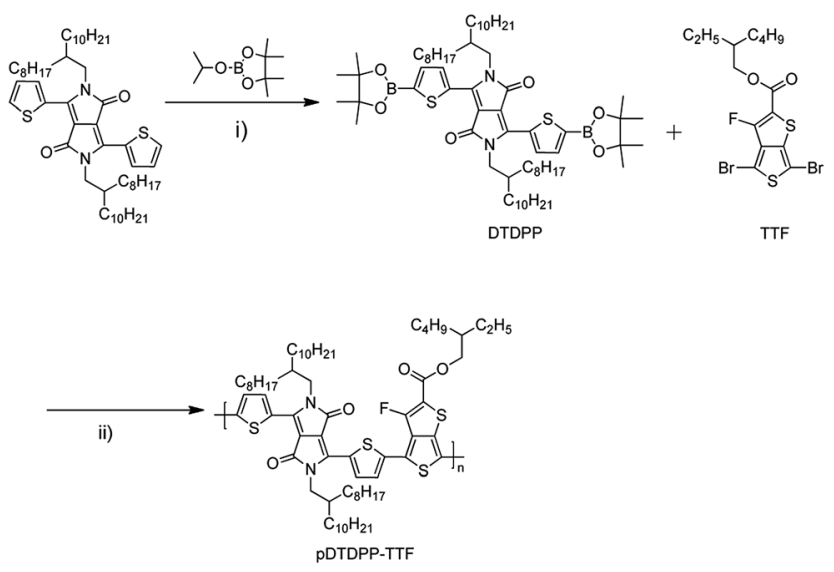

Scheme 1 Synthetic route for the copolymer pDTDPP-TTF. Reagents and conditions: (i) LDA, THF, $-25{ }^{\circ} \mathrm{C}$ under $\mathrm{N}_{2}, 2 \mathrm{~h}, 72 \%$; (ii) $\mathrm{Pd}_{2}(\mathrm{dba})_{3} /$ $\mathrm{P}(\text { o-tol) })_{3}, \mathrm{~K}_{3} \mathrm{PO}_{4}$, Aliquat 336, toluene $/ \mathrm{H}_{2} \mathrm{O}, 95{ }^{\circ} \mathrm{C}$ for $48 \mathrm{~h}, 50 \%$. processed OFET devices with both of the BG-BC and TG-BC configurations.

\section{Results and discussion}

\subsection{Monomers and polymer synthesis}

As shown in Scheme 1, pDTDPP-TTF was feasibly synthesized by conventional Suzuki cross-coupling polycondensation between 3,6-di(2-(4,4,5,5-tetramethyl-[1,3,2]dioxaborolan-2-yl) thien-5-yl)-2,5-di(2-decyltetradecyl)-pyrrolo[3,4-c]pyrrole-1,4dione (DTDPP) and 2-ethylhexyl-4,6-dibromo-3-fluorothieno $[3,4-b]$ thiophene-2-carboxylate (TTF), synthetic details can be found in the experimental section. After removing the impurities and low-molecular-weight oligomers of the resultant polymer by Sohxlet extraction (methanol, $(6 \mathrm{~h})$ acetone $(6 \mathrm{~h})$, and hexane $(6 \mathrm{~h})$ ), the residue was extracted with chlorobenzene to afford the target polymer with moderate number-average molecular weights $\left(M_{\mathrm{n}}=16 \mathrm{~kg} \mathrm{~mol}{ }^{-1}\right)$ and reasonable polydispersity indexes (PDIs $=2.2$ ), as listed in Table 1 . The polymer structure was characterized by a combination of ${ }^{1} \mathrm{H}$ NMR spectroscopy (as shown in ESI of Fig. S1†) as well as elemental analysis which was in agreement with the predicted values. The polymer pDTDDP-TTF showed reasonable solubility in common organic halogenated solvents, such as chloroform, chlorobenzene and 1,2-dichlorobenzene, likely due to the long alkyl branched side chains on the DPP and TTF repeat units.

\subsection{Thermal stability}

The thermal properties of pDTDPP-TTF were examined by differential scanning calorimetry (DSC) and thermogravimetric analysis (TGA) measurements. No clear thermal transitions within the range of 25 to $300{ }^{\circ} \mathrm{C}$ were detected from the DSC curves for the polymer. From the TGA curves in Fig. 1, the polymer pDTDPP-TTF exhibited good thermal stability with a decomposition temperature ( $5 \%$ weight loss) of $379^{\circ} \mathrm{C}$.

\subsection{Optical properties}

The UV-vis absorption spectra of pDTDPP-TTF in dilute chloroform solution and thin films are shown in Fig. 2 and the corresponding absorption properties are summarized in Table 1. In solution, pDTDPP-TTF exhibited a strong, broad and featureless absorption band spanning the visible to near infrared range (600 to $1100 \mathrm{~nm}$ ), arising from $\pi-\pi *$ transitions in the polymer backbone.

Moreover, at the maximum absorption wavelength of 830 $\mathrm{nm}$, pDTDPP-TTF exhibited a very high molar extinction coefficient of $\varepsilon=3.24 \times 10^{4} \mathrm{M}^{-1} \mathrm{~cm}^{-1}$. In comparison, the thin film UV-vis absorption spectra of pDTDPP-TTF was red-shifted by approximately $110 \mathrm{~nm}$. We attribute this shift to the longer wavelength region upon solidification to aggregation and solid state packing effects. The optical bandgap $\left(E_{\mathrm{g}}^{\mathrm{opt}}\right)$ of pDTDPPTTF was estimated to be $\sim 1.06 \mathrm{eV}$ from the absorption onset in the solid state. The ultra-low bandgap of pDTDPP-TTF is comparable or even lower than traditional single crystal silicon $(1.11 \mathrm{eV})$, due to the introduction of two electron-deficient units in the polymer backbone. To the best of our knowledge, the $E_{\mathrm{g}}$ is 
Table 1 Characteristics of the copolymer pDTDPP-TTF

\begin{tabular}{lllllll}
\hline Polymer & $M_{\mathrm{n}} / M_{\mathrm{w}}(\mathrm{kDa})$ & $\lambda_{\mathrm{abs}, \max }{ }^{a}(\mathrm{~nm})$ & $\lambda_{\text {onset }^{b}(\mathrm{~nm})}$ & $E_{\mathrm{g}}{ }^{c}(\mathrm{eV})$ & $\mathrm{HOMO}^{d}(\mathrm{eV})$ & $\mathrm{LUMO}^{e}(\mathrm{eV})$ \\
\hline pDTDPP-TTF & $16 / 36$ & $830(940)$ & 1166 & 1.06 & -5.43
\end{tabular}

${ }^{a}$ Measured in dilute $\mathrm{CHCl}_{3}$ solution and thin neat film (parentheses). ${ }^{b}$ Measured at film state. ${ }^{c} E_{\mathrm{g}}$ was calculated from the onsets of the UV-vis spectra in neat film, $E_{\mathrm{g}}=1240 / \lambda_{\text {onset. }}{ }^{d}$ HOMO was measured by cyclic voltammetry. ${ }^{e}$ Calculated from the equation LUMO $=\mathrm{HOMO}+E_{\mathrm{g}}$.

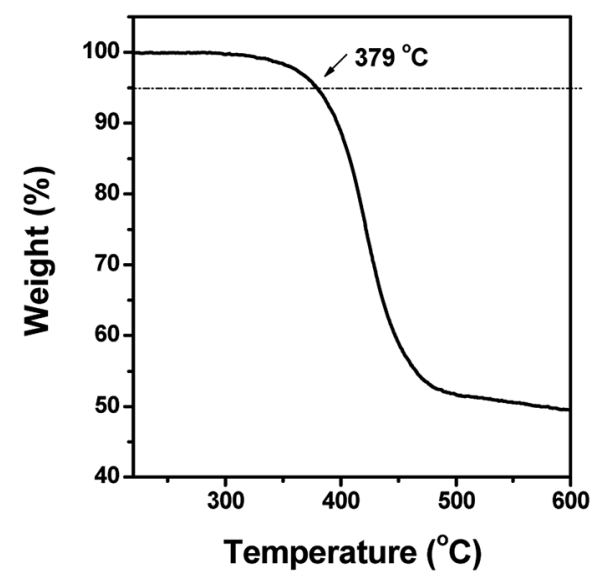

Fig. 1 TGA curve of the copolymer pDTDPP-TTF measured within the range from 25 to $600{ }^{\circ} \mathrm{C}$ at a heating rate of $20{ }^{\circ} \mathrm{C} \mathrm{min}-1$ under a nitrogen atmosphere.

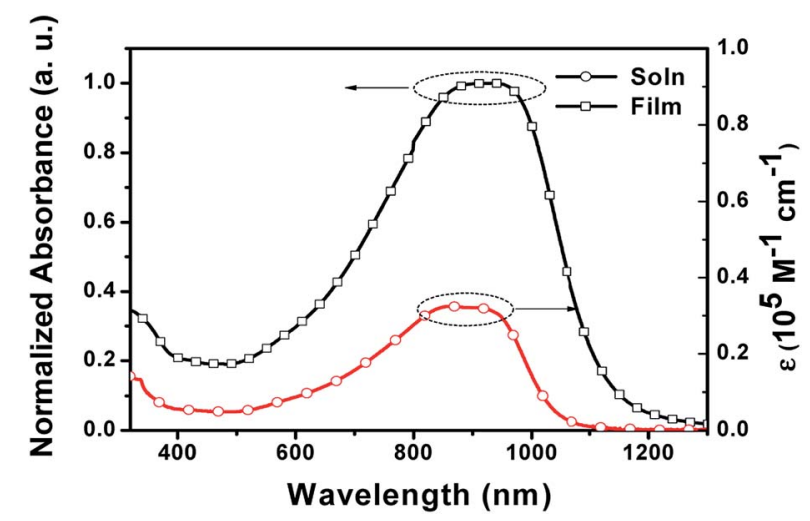

Fig. 2 UV-vis absorption spectra of pDTDPP-TTF in neat film (normalized, black) and in $10^{-5} \mathrm{M}$ of $\mathrm{CHCl}_{3}$ solution with molar absorptivity $(\varepsilon)$ (red).

among the lowest values ever reported for potential ambipolar semiconducting polymers in the literature..$^{30,31,35,36}$

\subsection{Film morphology}

Since film morphology and crystallinity are known to be key parameters for device performance, polymer thin films were analyzed using atomic force microscopy (AFM) and GIWAXS measurements. As depicted in the AFM images shown in Fig. 3(a), the root mean-square surface roughness (RMS) of pDTDPP-TTF thin films is $0.60 \mathrm{~nm}$ for $5 \mu \mathrm{m} \times 5 \mu \mathrm{m}$ scan area. XRD analysis was performed on PDTDPP-TTF thin films spin- casted from chloroform solution onto a Si wafer. As shown in Fig. 3(b), the GIWAXS pattern is fairly featureless compared to other high mobility polymer semiconductors. ${ }^{23,35}$ Only a single order preferentially in-plane alkyl stacking peak at $q=0.29 \AA^{-1}$ $(d=2.2 \mathrm{~nm})$ and a broad $\pi-\pi$ stacking peak in the out-of-plane direction at $q=1.71 \AA^{-1}(d=0.37 \mathrm{~nm})$ were observed.

\subsection{Electrochemical properties}

The electrochemical properties of PDTDPP-TTF were investigated by cyclic voltammetry (CV) in acetonitrile (MeCN)

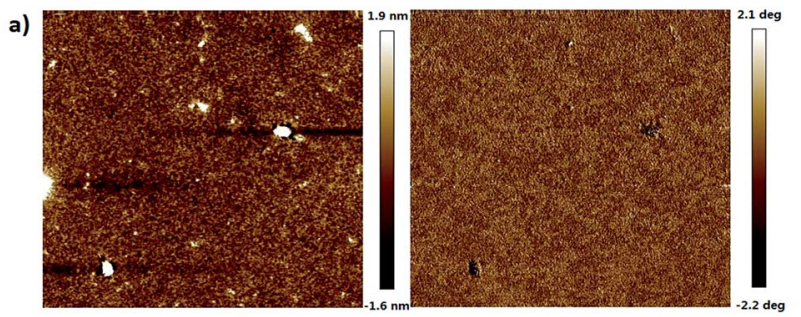

b)
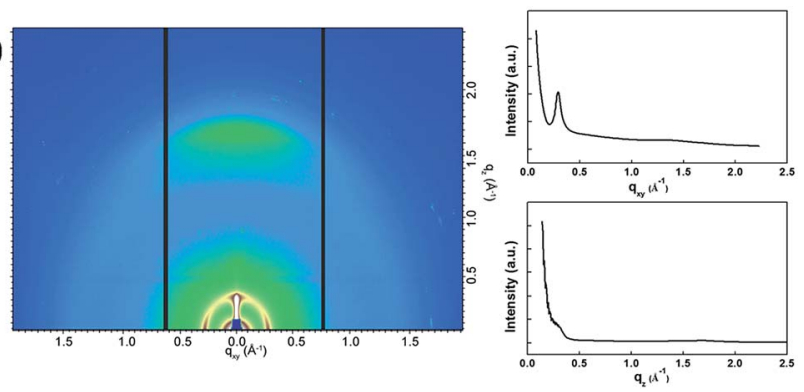

Fig. 3 (a) Topography (left) and phase (right) AFM images. Scale $5 \mu \mathrm{m}$ $\times 5 \mu \mathrm{m}$ (up) and (b) GIWAXS Pattern of pDTDPP-TTF thin film (bottom).

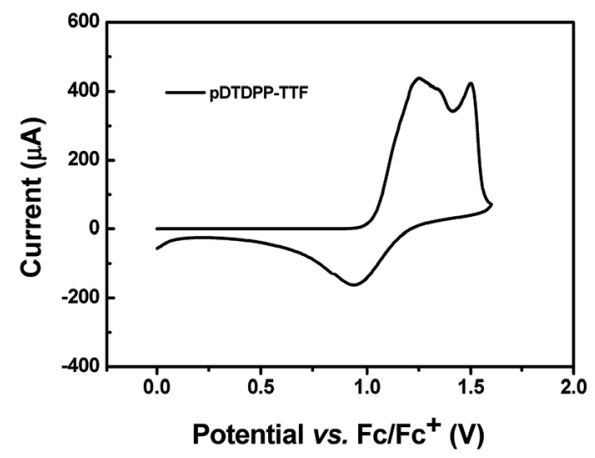

Fig. 4 Cyclic voltammetry of pDTDPP-TTF film in $0.1 \mathrm{~mol} \mathrm{~L}^{-1}$ $n-\mathrm{Bu}_{4} \mathrm{NPF}_{6}$ of acetonitrile solution, with a scan rate of $100 \mathrm{mV} \mathrm{s}{ }^{-1}$. 
solution. As shown in Fig. 4, the polymer exhibited quasireversible oxidation and undetectable reduction behavior. The onset of the oxidation potential $\left(E_{\text {onset }}\right)$ of pDTDPP-TTF occured at $1.06 \mathrm{eV}$, from which the HOMO energy level is calculated to be $-5.43 \mathrm{eV}$ based on the equation of HOMO $=-\left(4.4+E_{\text {onset }}\right){ }^{37}$

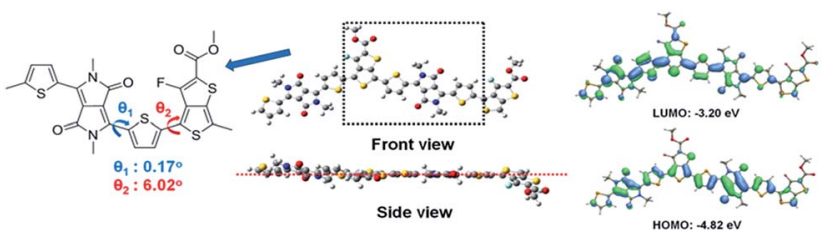

Fig. 5 DFT calculations on the optimized geometries and molecular orbitals for a DTDPP-TTF dimer (B3LYP/6-31G*).
The LUMO energy level can be obtained from the difference between optical bandgap $\left(E_{\mathrm{g}}\right)$ and HOMO using the equation $\mathrm{LUMO}=\left(\mathrm{HOMO}+E_{\mathrm{g}}\right)$, which was determined to be $-4.37 \mathrm{eV}$.

\subsection{Density functional theory calculation}

Fig. 5 shows the electron-state-density distribution of the highest occupied molecular orbital (HOMO) and the lowest unoccupied molecular orbital (LUMO) of geometrically optimized structures determined by density functional theory $(\mathrm{DFT})^{38}$ at the B3LYP ${ }^{39}$ hybrid functional level using a 6-31G* basis set. In order to simplify the calculations and minimize the computational cost, the polymer model was simplified by dimeric systems and the long alkyl substituted side chains in the polymer molecular skeleton were replaced by methyl groups. As shown in Fig. 5, two dihedral angles were evaluated, where the first between the DPP unit and the adjacent a)

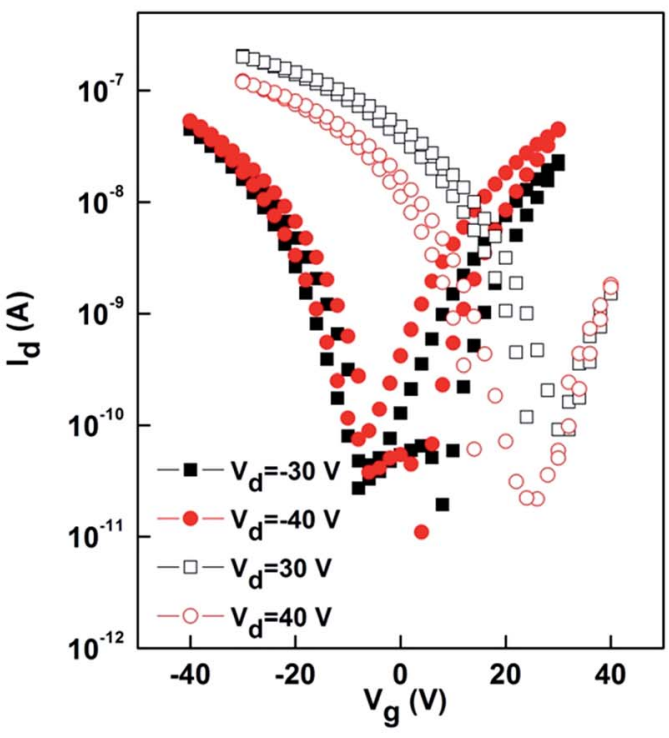

b)

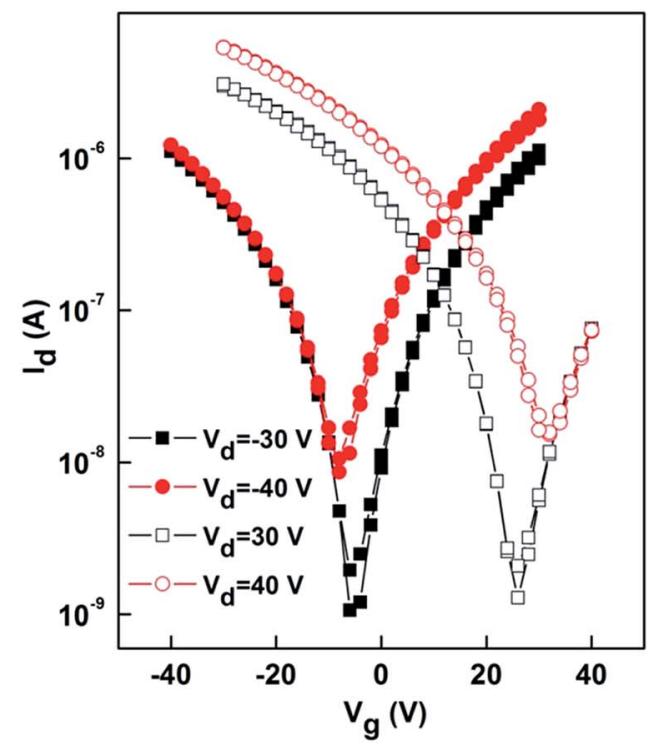

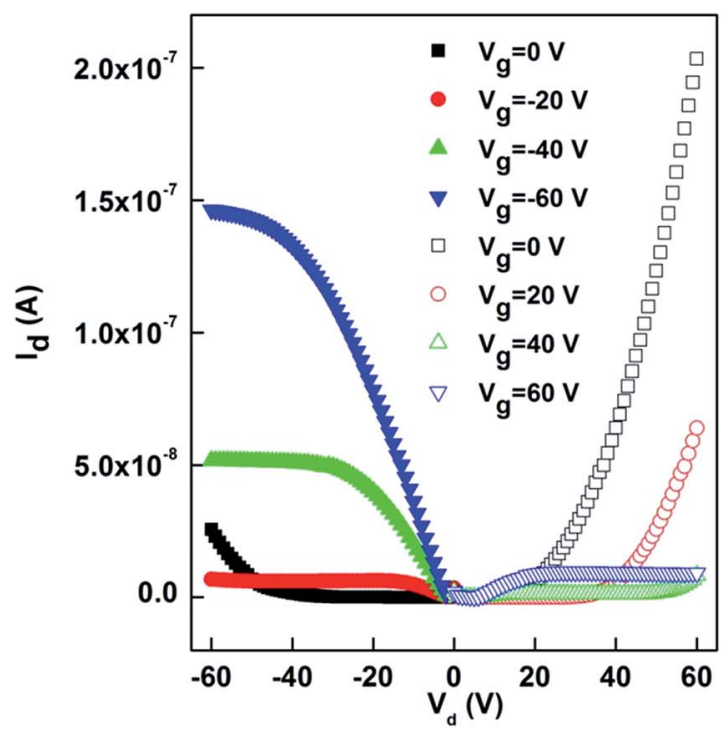

Fig. 6 Typical transfer and output plot of pDTDPP-TTF OFETs in (a) BG-BC and (b) TG-BC device structures. The devices were annealed at $180{ }^{\circ} \mathrm{C}$. For the BG-BC devices the solvent for pDTDPP-TTF is chloroform while for the TG-BC devices the solvent is 1,2-dichlorobenzene. 
Table 2 Summary of field-effect mobilities for the copolymer over 4 devices

\begin{tabular}{lllllll}
\hline & \multicolumn{2}{l}{ Mobility $\left(\mathrm{cm}^{2} \mathrm{~V}^{-1} \mathrm{~s}^{-1}\right)$} & & & \multicolumn{2}{c}{ Threshold voltage $(\mathrm{V})$} \\
\cline { 2 - 3 } Devices & Hole & & Hole & Electron & On/off ratio \\
\hline (BG-BC) & $(1.85 \pm 0.15) \times 10^{-4}$ & $(3.55 \pm 0.34) \times 10^{-5}$ & & $-7.4 \pm 3.2$ & $24.9 \pm 9.1$ & $(7.8 \pm 1.2) \times 10^{3}$ \\
(TG-BC) & $(6.17 \pm 0.59) \times 10^{-3}$ & $(3.13 \pm 0.50) \times 10^{-3}$ & & $-9.0 \pm 0.5$ & $23.2 \pm 3.7$ & $(1.5 \pm 0.7) \times 10^{3}$
\end{tabular}

thiophene ring $\left(\theta_{1}\right)$, and the second between thiophene and the adjacent TTF unit $\left(\theta_{2}\right)$. The $\theta_{1}$ and $\theta_{2}$ values obtained from the dimmers of pDTDPP-TTF were calculated to be $0.17^{\circ}$ and $6.02^{\circ}$, respectively. From the side view of the optimized geometry, a relatively planar backbone structure is predicted for pDTDPPTTF, which is in agreement with other dithienyl-DPP containing copolymers reported in the literature..$^{\mathbf{1 0 , 1 5 , 2 1}}$ It is implied that pDTDPP-TTF may exhibit strong $\pi-\pi$ stacking due to the planarity of the polymer backbone.

Both the HOMO and LUMO energy levels were mainly delocalized through the entire molecular skeleton. The calculated HOMO and LUMO energy levels for the dimer of pDTDPP-TTF were -4.82 and $-3.20 \mathrm{eV}$, respectively. It should be noted that slight differences between the absolute values of the computationally derived and experimentally determined results is acceptable to exist and can be attributed to the inherent limitations of DFT to accurately predict the behavior of organic semiconducting polymer as well as the intrinsic discrepancy in the dimeric and polymeric molecular skeleton. ${ }^{\mathbf{4 0}}$ However, both the DFT and CV methods revealed similar trends in the energy levels suggesting the low bandgap nature and the potential ambipolar charge transport behaviour of pDTDPP-TTF polymer.

\subsection{Field-effect transistor characteristics}

The appropriate HOMO and LUMO energy level alignment of pDTDPP-TTF encouraged us to investigate the charge transport properties of the polymer material. Therefore, OFET devices with a bottom-gate, bottom-contact (BG-BC) configuration were initially fabricated. Gold $(\mathrm{Au})$ source-drain electrodes were prepared by photolithography on the $\mathrm{SiO}_{2} / \mathrm{Si}^{++}$substrates. Then the substrates were passivated with DTS before the polymer films were deposited onto the substrates by spin-coating from a chloroform-based solution. Fig. 6(a) shows the representative transfer and output characteristics of BG-BC OFET devices based on spin-coated films of pDTDPP-TTF after annealing at $180{ }^{\circ} \mathrm{C}$. It is seen that pDTDPP-TTF exhibited typical V-shaped ambipolar behavior. The device performance data are summarized in Table 2. Though the BG-BC devices with $\mathrm{SiO}_{2}$ as dielectric demonstrated ambipolar transport, the mobility for both holes and electrons was rather low. The average hole and electron mobilities were measured to be $1.85 \times 10^{-4}$ and $3.55 \times 10^{-5} \mathrm{~cm}^{2} \mathrm{~V}^{-1}$ $\mathrm{s}^{-1}$, respectively. To further improve the OFET performance, topgate bottom-contact (TG-BC) devices using PMMA as dielectric were then fabricated, since PMMA is generally known as a good dielectric for efficient electron transport. 1,2-Dichlorobenzene was selected as the solvent because the high-boiling point solvent may yield higher performance. ${ }^{41}$ In the PMMA-based OFETs, balanced ambipolar transport with significantly enhanced performance was obtained. The extracted hole and electron mobilities was measured to be $6.17 \times 10^{-3}$ and $3.13 \times 10^{-3} \mathrm{~cm}^{2}$ $\mathrm{V}^{-1} \mathrm{~s}^{-1}$, respectively, which was over one order of magnitude higher than the values in the BG-BC devices. It is expected that further enhanced mobility is allowed to achieve by optimization of the HOMO/LUMO energy levels alignment with the work function of electrodes.

\section{Conclusions}

In conclusion, we have designed and synthesized a new ultralow bandgap polymer material pDTDPP-TTF with ambipolar charge transport properties via a Suzuki cross-coupling polycondensation reaction. Molecular design strategy on the introduction of $\mathrm{A}_{1}-\mathrm{A}_{2}$ type monomer has been successfully conducted to significantly lower the LUMO level of DPP based polymer to as low as $-4.37 \mathrm{eV}$, which is potentially suitable for efficient electron injection and transport. The polymer exhibited a broad and featureless absorption spectrum ranging from visible to up to $1300 \mathrm{~nm}$, with a narrow bandgap of $\sim 1.06 \mathrm{eV}$. Ambipolar transport behaviour has been achieved in solutionprocessed OFET devices based on PDTDPP-TTF active layers in both the BG-BC and TG-BC configurations. Relatively balanced ambipolar charge transport with a hole and electron mobility of $6.17 \times 10^{-3} \mathrm{~cm}^{2} \mathrm{~V}^{-1} \mathrm{~s}^{-1}$ and $3.13 \times 10^{-3} \mathrm{~cm}^{2} \mathrm{~V}^{-1}$ $\mathrm{s}^{-1}$ respectively has been obtained when PMMA was used as dielectric. The results indicate the potential of $\mathrm{A}_{1}-\mathrm{A}_{2}$ type copolymers as active materials for polymer solar cells, near infrared photodetectors and/or other organic electronic devices. And the synthetic route on the introduction of an additional acceptor unit to construct $\mathrm{A}_{1}-\mathrm{A}_{2}$ type polymer provides molecular design strategy to lower the LUMO energy levels for balanced ambipolar ultra-low bandgap semiconducting polymers.

\section{Experimental}

\subsection{Materials}

All of the reagents were obtained from commercial suppliers and used without further purification, unless stated otherwise. THF and toluene were dried over $\mathrm{Na}$ /benzophenoneketyl and freshly distilled prior to use. 3,6-Dithien-2-yl-2,5-di(2-decyltetradecanyl)-pyrrolo[3,4-c]pyrrole-1,4-dione (M1) was prepared according to established literature procedures, ${ }^{\mathbf{4 2 , 4 3}}$ and the monomer TTF (M2) was purchased from Suna Tech Inc. 


\subsection{Measurements and characterization}

${ }^{1} \mathrm{H}$ NMR spectra was measured on a Bruker DRX-400 spectrometer in $\mathrm{CDCl}_{3}$ using tetramethylsilane as the internal reference. Mass spectra were measured on Bruker autoflex matrix-assisted laser desorption/ionization time-of-flight (MALDI-TOF). Elemental analysis of carbon, hydrogen and nitrogen was performed on a Vario EL III microanalyzer. UVvis absorption spectra were recorded on a Shimadzu UV-2500 recording spectrophotometer. Differential scanning calorimetry (DSC) was performed on a NETZSCH DSC 200 PC unit at a heating rate of $10{ }^{\circ} \mathrm{C} \mathrm{min}{ }^{-1}$ from 25 to $300{ }^{\circ} \mathrm{C}$, under argon. Thermogravimetric analysis (TGA) was undertaken with a NETZSCH STA 449C instrument. The thermal stability of the samples under a nitrogen atmosphere was determined by measuring their weight loss at a heating rate of $20{ }^{\circ} \mathrm{C} \mathrm{min}{ }^{-1}$ from 25 to $600{ }^{\circ} \mathrm{C}$. The electrochemical cyclic voltammetry was conducted on a CHI voltammetric analyzer, in a $0.1 \mathrm{~mol} \mathrm{~L}^{-1}$ acetonitrile solution of tetrabutylammonium hexafluorophosphate $\left(n-\mathrm{Bu}_{4} \mathrm{NPF}_{6}\right)$ at a potential scan rate of $100 \mathrm{mV} \mathrm{s}^{-1}$, along with an $\mathrm{Ag} / \mathrm{AgCl}$ reference electrode and a platinum wire counter electrode. The polymer sample was coated on the platinum sheet of working electrode. The reference electrode was checked versus ferrocene as internal standard as recommended by IUPAC. Atomic force microscopy (AFM) was conducted on SPA300HV in tapping mode using an SPI3800 controller, Seiko Instruments Industry, Co., Ltd. GIWAXS measurements were done in a helium environment at the Advanced Light Source beamline 7.3.3. ${ }^{44}$ Sample-todetector distance was $300 \mathrm{~mm}$, calibrated by an AgB standard, and data was collected at an incident angle of $0.14^{\circ}$. The organic field effect transistor (OFET) device characteristics were measured using an Agilent 4155B semiconductor parameter analyzer. The mobility was determined in the saturation regime by using the equation $I_{\mathrm{DS}}=\left(\mu W C_{\mathrm{i}} / 2 L\right)\left(V_{\mathrm{G}}-\right.$ $\left.V_{\mathrm{T}}\right) 2$, where $I_{\mathrm{DS}}$ is the drain-source current, $\mu$ the field-effect mobility, $W$ the channel width, $L$ the channel length, $C_{\mathrm{i}}$ the capacitance per unit area of the gate dielectric layer and $V_{\mathrm{T}}$ the threshold voltage.

\subsection{Device fabrication and measurements}

Bottom-gate, bottom-contact (BG-BC) devices were fabricated on $\mathrm{SiO}_{2} / \mathrm{Si}^{++}$substrates. The source/drain electrodes were patterned by standard photolithography. The substrates were then passivated with DTS by immersing them into the solution (DTS : toluene $=1: 1000)$. Top-gate, bottom-contact (TG-BC) devices were fabricated on glass substrates. Gold source and drain electrodes $(20 \mathrm{~nm})$ were patterned by standard photolithography with a channel length $L=20 \mu \mathrm{m}$ and width $W=1$ $\mathrm{mm}$. All the spin-coating processes were done in nitrogen filled glovebox. The polymer was spin-coated from a chloroform solution or 1,2-dichlorobenzene $\left(5 \mathrm{mg} \mathrm{mL}^{-1}\right)$ solution at 1000 rpm. For TG-BC devices, a $500 \mathrm{~nm}$ thick PMMA was spin-coated on the polymer film and annealed at $80{ }^{\circ} \mathrm{C}$ for $30 \mathrm{~min}$. The PMMA was dissolved in an orthogonal solvent of $n$-butyl acetate which prevents dissolving the underneath semiconducting layers. Finally, a $20 \mathrm{~nm}$ thick gold gate electrode was deposited by shadow-mask evaporation. Devices were annealed in glovebox for $10 \mathrm{~min}$ at a temperature of $180{ }^{\circ} \mathrm{C}$ before the electrical test.

\subsection{Synthesis of pDTDPP-TTF}

To a $50 \mathrm{~mL}$ Schlenk tube, M1 (327 mg, $0.3 \mathrm{mmol}$ ), 3-fluorothieno[3,4- $b]$ thiophene-2-carboxylate (TTF) M2 (155 mg, 0.3 mmol $), \mathrm{Pd}_{2}(\mathrm{dba})_{3}(9 \mathrm{mg}, 0.01 \mathrm{mmol}), \mathrm{P}(o-\text { tol })_{3}$ (12 mg, 0.04 $\mathrm{mmol}$ ) and 2 drops Aliquant 336 were added. The tube was then charged with Ar through a freeze-pump-thaw cycles for three times. Then $\mathrm{K}_{3} \mathrm{PO}_{4}(425 \mathrm{mg}, 2 \mathrm{mmol})$ in toluene $(5 \mathrm{~mL})$ with demineralized water $(1 \mathrm{~mL})$ were added under an argon atmosphere. The mixture was stirred for $48 \mathrm{~h}$ at $95{ }^{\circ} \mathrm{C}$. After cooling down to room temperature, the highly viscous black gel was precipitated to $200 \mathrm{~mL}$ of methanol containing $20 \mathrm{~mL}$ of distilled water and then stirred for $3 \mathrm{~h}$. The resulting polymer was collected by filtration and washed with methanol $(100 \mathrm{~mL})$ and acetone $(100 \mathrm{~mL})$. The crude product was further purified by Soxhlet extraction with methanol $(6 \mathrm{~h})$, acetone $(6 \mathrm{~h})$, hexane (6 h), and finally CB (6 h). A free-standing film was obtained after removing CB solvent (245.8 $\mathrm{mg}, 0.21 \mathrm{mmol}, 70 \%$ yield).

${ }^{1} \mathrm{H}$ NMR ( $\left.\mathrm{CDCl}_{3}, 400 \mathrm{MHz}\right), \delta(\mathrm{ppm}): 9.17$ (br, 2H), $7.26(\mathrm{br}$, 2H), 4.35 (br, 6H), 1.9 (br, 3H), 1.44-1.02 (br, 72H), 0.81 (br, $18 \mathrm{H}$ ); anal. calcd: C, 70.72; H, 8.86; N, 2.39\% found: C, 70.60; H, 8.66; N, 2.35\%. GPC: $M_{\mathrm{n}}=16 \mathrm{kDa}, M_{\mathrm{w}}=36 \mathrm{kDa}, \mathrm{PDI}=2.2$.

\section{Acknowledgements}

The authors thank the National Natural Science Foundation of China (21304047), Natural Science Foundation of Jiangshu Province (BK20130919, 13KJB430017), Research Fund for the Doctoral Program of Higher Education (20133221120015) and Postgraduate Innovation Foundation of Jiangsu Province (2014, KYZZ_0226) for financial support. We acknowledge the Advanced Light Source, which is supported by the Director, Office of Science, Office of Basic Energy Sciences, of the U.S. Department of Energy under Contract No. DE-AC02$05 \mathrm{CH} 11231$.

\section{Notes and references}

1 R. A. Street, Thin-Film Transistors, Adv. Mater., 2009, 21, 2007.

2 B. Crone, A. Dodabalapur, Y.-Y. Lin, R. W. Filas, Z. Bao, A. LaDuca, R. Sarpeshkar, H. E. Katz and W. Lin, Nature, 2000, 403, 521.

3 G. H. Gelinck, H. Edzer, A. Huitema, E. V. Veenendaal, E. van Cantatore, L. Schrijnemakers, J. B. P. H. van der Putten, T. C. T. Geuns, M. Beenhakkers, J. B. Giesbers, B.-H. Hiusman, E. J. Meijer, E. M. Benito, F. J. Touwslager, A. W. Marsman, B. J. E. van Rens and D. M. De Leeuw, Nat. Mater., 2004, 3, 106.

4 A. Lorenzoni, M. Muccini and F. Mercuri, $R S C A d v .$, 2015, 5, 11797. 
5 P. F. Baude, D. A. Ender, M. A. Haase, T. W. Kelley, D. V. Muyres and S. D. Theiss, Appl. Phys. Lett., 2003, 82, 3964.

6 B. Crone, A. Dodabalapur, A. Gelperin, L. Torsi, H. E. Katz, A. J. Lovinger and Z. Bao, Appl. Phys. Lett., 2001, 78, 2229.

7 Z. H. Sun, J. H. Li, C. M. Liu, S. H. Yang and F. Yan, Adv. Mater., 2011, 23, 3648.

8 P. Lin and F. Yan, Adv. Mater., 2012, 24, 34.

9 H.-R. Tseng, H. Phan, C. Luo, M. Wang, L. A. Perez, S. N. Patel, L. Ying, E. J. Kramer, T.-Q. Nguyen, G. C. Bazan and A. J. Heeger, Adv. Mater., 2014, 26, 2993.

10 H.-J. Yun, S.-J. Kang, Y. Xu, S. O. Kim, Y.-H. Kim, Y.-Y. Noh and S.-K. Kwon, Adv. Mater., 2014, 26, 7300.

11 Z. Chen, H. Lemke, S. Albert-Seifried, M. Caironi, M. M. Nielsen, M. Heeney, W. Zhang, I. McCulloch and H. Sirringhaus, Adv. Mater., 2010, 22, 2371.

12 H. Klauk, U. Zschieschang, J. Pflaum and M. Halik, Nature, 2007, 445, 745.

13 J. Zaumseil, R. H. Friend and H. Sirringhaus, Nat. Mater., 2006, 5, 69.

14 L. Bürgi, M. Turbiez, R. Pfeiffer, F. Bienewald, H.-J. Kirner and C. Winnewisser, Adv. Mater., 2008, 20, 2217.

15 Z. Chen, M. J. Lee, R. S. Ashraf, Y. Gu, S. Albert-Seifried, M. M. Nielsen, B. Schroeder, T. D. Anthopoulos, M. Heeney, I. McCulloch and H. Sirringhaus, Adv. Mater., 2012, 24, 647.

16 C. B. Nielsen, M. Turbiez and I. McCulloch, Adv. Mater., 2013, 25, 1859.

17 Y. Li, P. Sonar, L. Murphy and W. Hong, Energy Environ. Sci., 2013, 6, 1684.

18 H. N. Tsao, D. M. Cho, I. Park, M. R. Hansen, A. Mavrinskiy, D. Y. Yoon, R. Graf, W. Pisula, H. W. Spiess and K. Müllen, J. Am. Chem. Soc., 2011, 133, 2605.

19 X. Chen, B. Liu, Y. Zou, W. Tang, Y. Li and D. Xiao, RSC Adv., 2012, 2, 7439.

20 C. Guo, W. Hong, H. Aziz and Y. Li, Reviews in Advanced Sciences and Engineering, 2012, 1, 200.

21 I. Kang, H.-J. Yun, D. S. Chung, S.-K. Kwon and Y.-H. Kim, J. Am. Chem. Soc., 2013, 135, 14896.

22 B. Sun, W. Hong, Z. Yan, H. Aziz and Y. Li, Adv. Mater., 2014, 26, 2636.

23 J. Lee, A.-R. Han, H. Yu, T. J. Shin, C. Yang and J. H. Oh, J. Am. Chem. Soc., 2013, 135, 9540.

24 O. Wallquist and R. Lenz, Macromol. Symp., 2002, 187, 617. 25 A. B. Tamayo, M. Tantiwiwat, B. Walker and T.-Q. Nguyen, J. Phys. Chem. C, 2008, 112, 15543.
26 H. F. Feng, W. F. Fu, L. J. Li, Q. C. Yu, H. Lu, J. H. Wan, M. M. Shi, H. Z. Chen, Z. A. Tan and Y. F. Li, Org. Electron., 2014, 15, 2575.

27 Y. Kim, C. E. Song, E.-J. Ko, D. Kim, S.-J. Moon and E. Lim, RSC Adv., 2015, 5, 4811.

28 H. Lin, W. Lee and W. Chen, J. Mater. Chem., 2012, 22, 2120.

29 J. S. Lee, S. K. Son, S. Song, H. Kim, D. R. Lee, K. Kim, M. J. Ko, D. H. Choi, B. Kim and J. H. Cho, Chem. Mater., 2012, 24, 1316.

30 J. D. Yuen, J. Fan, J. Seifter, B. Lim, R. Hufschmid, A. J. Heeger and F. Wudl, J. Am. Chem. Soc., 2011, 133, 20799.

31 M. Shahid, T. McCarthy-Ward, J. Labram, S. Rossbauer, E. B. Domingo, S. E. Watkins, N. Stingelin, T. D. Anthopoulos and M. Heeney, Chem. Sci., 2012, 3, 181. 32 H. Bronstein, Z. Chen, R. S. Ashraf, W. Zhang, J. Du, J. R. Durrant, P. S. Tuladhar, K. Song, S. E. Watkins, Y. Geerts, M. M. Wienk, R. A. J. Janssen, T. Anthopoulos, H. Sirringhaus, M. Heeney and I. McCulloch, J. Am. Chem. Soc., 2011, 133, 3272.

33 H. Yan, Z. Chen, Y. Zheng, C. Newman, J. R. Quinn, F. Dötz, M. Kastler and A. Facchetti, Nature, 2009, 457, 679.

34 S. Cho, J. Lee, M. Tong, J. H. Seo and C. Yang, Adv. Funct. Mater., 2011, 21, 1910.

35 A. J. Kronemeijer, E. Gili, M. Shahid, J. Rivnay, A. Salleo, M. Heeney and H. Sirringhaus, Adv. Mater., 2012, 24, 1558.

36 A. R. Mohebbi, J. Yuen, J. Fan, C. Munoz, M. Wang, R. S. Shirazi, J. Seifer and F. Wudl, Adv. Mater., 2011, 23, 4644.

37 H. J. Chen, Y. L. Guo, X. N. Sun, D. Gao, Y. Q. Liu and G. Yu, J. Polym. Sci., Part A: Polym. Chem., 2013, 51, 2208.

38 M. J. Frisch, et al., Gaussian 09, Revision A.2, Gaussian Inc, Wallingford CT, 2009.

39 A. D. Becke, J. Chem. Phys., 1993, 98, 5648.

40 C. Risko, M. D. McGehee and J.-L. Bredas, Chem. Sci., 2011, 2, 1200.

41 J.-F. Chang, B. Sun, S. W. Brieby, M. M. Nielsen, T. I. Solling, M. Giles, I. McCulloch and H. Sirringhaus, Chem. Mater., 2004, 16, 4772.

42 D. Cortizo-Lacalle, S. Arumugam, S. Elmasly, A. Kanibolotsky, N. Findlay, A. Inigo and P. Skabara, J. Mater. Chem., 2012, 22, 11310.

43 S. Wang, J. Yang, Z. Zhang, Y. Hu, X. Cao, H. Li, Y. Tao, Y. Li and W. Huang, RSC Adv., 2015, 5, 68192.

44 A. Hexemer, W. Bras, J. Glossinger, E. Schaible, E. Gann, R. Kirian, A. MacDowell, M. Church, B. Rude and H. Padmore, J. Phys.: Conf. Ser., 2010, 247, 012007. 